UDC 517.5

\title{
SPLITTING OF SOME SPACES OF ANALYTIC FUNCTIONS
}

\author{
V.N. DILNYI
}

\begin{abstract}
For the Paley-Wiener space and the weighted Hardy spaces in the half-plane we consider problems on splitting a function into a sum of two, each being "large" only in their domain. For the first space the problem is solved completely, for the second we obtain sufficient conditions of solvability.
\end{abstract}

Keywords: weighted Hardy space, Paley-Wiener theorem, angular boundary values, splitting

Mathematics Subject Classification: 2010: 30D15, 30H10

\section{INTRODUCTION}

We denote by $W_{\sigma}^{p}, 1 \leqslant p \leqslant 2, \sigma>0$, the Paley-Wiener space, i.e., the space of entire functions $f$ of exponential type $\leqslant \sigma$ belonging to $L^{p}(\mathbb{R})$. Space $W_{\sigma}^{p}$ can be defined (cf. [1]) as the space of entire functions satisfying the condition $A(0 ; 2 \pi)$, where

$$
A(\alpha, \beta):=\sup _{\varphi \in(\alpha, \beta)}\left\{\int_{0}^{+\infty}\left|f\left(r e^{i \varphi}\right)\right|^{p} e^{-p \sigma r|\sin \varphi|} d r\right\}^{1 / p}<+\infty .
$$

Spaces $W_{\sigma}^{p}, 1 \leqslant p \leqslant 2, \sigma>0$, are Banach ones with the norm $\|f\|:=A(0 ; 2 \pi)$. The next statement plays a fundamental role in the theory of Paley-Wiener spaces (see [2]).

Theorem (P.-W. theorem). Space $W_{\sigma}^{2}$ coincides with the space of functions represented as

$$
f(z)=\frac{1}{\sqrt{2 \pi}} \int_{-\sigma}^{\sigma} \varphi(t) e^{i t z} d t, \quad \varphi \in L^{2}(-\sigma, \sigma) .
$$

We denote by $H_{\sigma}^{p}\left(\mathbb{C}_{+}\right), 1 \leqslant p<+\infty, \sigma \geqslant 0$, the space of analytic in $\mathbb{C}_{+}=\{z: \operatorname{Re} z>0\}$ functions obeying the condition $A\left(-\frac{\pi}{2} ; \frac{\pi}{2}\right)<+\infty$. Certain properties of this space were studied by B.V. Vinnitskii and the author (see [6], [7], [18]). By $H^{p}\left(\mathbb{C}_{+}\right), 1 \leqslant p<+\infty$, we indicate the Hardy space of analytic in $\mathbb{C}_{+}$functions $f$ satisfying

$$
\|f\|^{p}=\sup _{x>0}\left\{\int_{-\infty}^{+\infty}|f(x+i y)|^{p} d y\right\}<+\infty .
$$

A.M. Sedletskii showed [10] that in the case $\sigma=0$ the space $H_{\sigma}^{p}\left(\mathbb{C}_{+}\right)$coincides with Hardy space and hence we can regard $H_{\sigma}^{p}\left(\mathbb{C}_{+}\right)$as a weighted Hardy space. It is also easy to see that $H_{\sigma}^{p}\left(\mathbb{C}_{+}\right)$is an analogue of Paley-Wiener space $W_{\sigma}^{p}$ of entire functions on a half-space. The properties of Hardy spaces are exposed in quite a details in [9], [15]. A singular boundary

V.N. Dilnyi, The SPlitting of Some spaces of analytic functions.

(C)DiLNYI V.N. 2014.

Submitted March 17, 2014. 
function $h$ of a function $\psi \in H_{\sigma}^{p}\left(\mathbb{C}_{+}\right)$is determined up to an additive constant and the values are the continuity points by the identity

$$
h\left(t_{2}\right)-h\left(t_{1}\right)=\lim _{x \rightarrow 0+} \int_{t_{1}}^{t_{2}} \log |\psi(x+i y)| d y-\int_{t_{1}}^{t_{2}} \log |\psi(i y)| d y .
$$

The singular boundary function is non-increasing and $h^{\prime}(t)=0$ for a.e. $t \in \mathbb{R}$.

\section{PROBLEMS}

R.S. Yulmukhametov in [3], [4] considered the problem on splitting each function in PaleyWiener space into the product of two functions for the case when $\varphi$ in the representation (1) is infinitely differentiable. Yu.I. Lyubarskii in [25] studied the splitting of functions with a triangle indicator diagram. We consider similar problems on splitting functions in Paley-Wiener space into the sum of two functions each of those, in some sense, inherits the properties of the original function.

The problems we consider in what follows are motivated by the studies $[8,14]$, where the complete description of cyclic functions in the space $H_{\sigma}^{2}\left(\mathbb{C}_{+}\right)$was obtained. It happened that there are qualitative differences in comparison with the non-weighted case $H^{p}\left(\mathbb{C}_{+}\right)$. For the further advancing in this direction, namely, for the description of all subspaces in space $H_{\sigma}^{2}\left(\mathbb{C}_{+}\right)$ translation invariant w.r.t. the shift operator, a key role is played by the following statement [7], whose proof we know only in the case $p \in(1 ; 2]$.

Theorem A1. A function $\widetilde{f}_{1}: i \mathbb{R} \rightarrow \mathbb{C}$, such that $\widetilde{f}_{1}(i y) e^{-\sigma|y|} \in L^{p}(-\infty ;+\infty)$ is the angular boundary function of a function $\tilde{f} \in H_{\sigma}^{p}\left(\mathbb{C}_{+}\right), 1<p \leqslant 2$, if and only if there exists a function $\widetilde{f}_{2}$ satisfying the conditions

a) $\widetilde{f}_{2} \in H_{2 \sigma}^{p}\left(\mathbb{C}_{+}\right)$;

b) $\widetilde{f}_{3}(i y):=e^{-\sigma y} \widetilde{f}_{1}(i y)+\widetilde{f}_{2}(i y) \in L^{p}(-\infty ; 0)$;

c) $\quad \int_{0}^{+\infty} \widetilde{f}_{1}(i v) e^{-\sigma t} e^{i \tau v} d v+\frac{1}{i} \int_{0}^{+\infty} \widetilde{f}_{2}(u) e^{\tau u} d u+\int_{-\infty}^{0} \widetilde{f}_{3}(i v) e^{i \tau v} d v=0$ for a.e. $\tau \in(-\infty ; 0]$.

In the case $\sigma=0$ (then $\widetilde{f}_{2} \equiv 0$ ) the conditions a), b) are trivial, while condition c) becomes $" \int_{-\infty}^{+\infty} \widetilde{f}_{1}(i v) e^{i \tau v} d v=0$ for a.e. $\tau \in(-\infty ; 0] . "$ Then this theorem coincides with one theorem by Paley-Wiener (see [15]). For the case $p=1$ in [7] the following statement was obtained.

Theorem A2. Function $\widetilde{f}_{1}: i \mathbb{R} \rightarrow \mathbb{C}$, such that $\widetilde{f}_{1}(i y) e^{-\sigma|y|} \in L^{p}(-\infty ;+\infty)$ is the angular boundary function of a function $\widetilde{f} \in H_{\sigma}^{1}\left(\mathbb{C}_{+}\right)$, if there exists a function $\widetilde{f}_{2}$ satisfying a), b), c) of the previous theorem and

d) function $\widetilde{f}_{2}$ admits the splitting $\widetilde{f}_{2}=\widetilde{f}_{5}-\widetilde{f}_{4}$, where $\widetilde{f}_{4}(z) e^{-i \sigma z} \in H_{\sigma}^{1}\left(\mathbb{C}_{+}\right)$, $\widetilde{f}_{5}(z) e^{i \sigma z} \in H_{\sigma}^{1}\left(\mathbb{C}_{+}\right)$.

We do not know whether this theorem is valid without condition d).

In studying one equation of convolution type [24] it was shown that the absence of nontrivial solution to this equation is equivalent to an analogue of Theorem A2 as $p=1$. The main difference of this analogue and our formulation is that additionally one can regard $\widetilde{f}_{2}$ as the product of two functions in $H_{\sigma}^{2}\left(\mathbb{C}_{+}\right)$. We do not know whether this addition is essential. i.e., whether each function $f \in H_{2 \sigma}^{1}\left(\mathbb{C}_{+}\right)$admits the splitting into the product of two functions in $H_{\sigma}^{2}\left(\mathbb{C}_{+}\right)$. But such splitting, in addition to the splitting $H_{\sigma}^{2}\left(\mathbb{C}_{+}\right)=e^{i \sigma z} H^{2}\left(\mathbb{C}_{+}\right)+e^{-i \sigma z} H^{2}\left(\mathbb{C}_{+}\right)+$ $W_{\sigma}^{2}$ obtained by B. Vinnitskii allows us to consider the following problem. 
Problem 1. Whether each function $f \in W_{\sigma}^{p}, 1 \leqslant p \leqslant 2$, admits the splitting $f=f_{2}-f_{3}$ with an entire function $f_{2}$ obeying condition $B(0 ; \pi)$, where

$$
B(\hat{\alpha}, \hat{\beta}): \sup _{\varphi \in(\hat{\alpha}, \hat{\beta})}\left\{\int_{0}^{+\infty}\left|f\left(r e^{i \varphi}\right)\right|^{p} d r\right\}^{1 / p}<+\infty,
$$

and $f_{3}$ obeying condition $B(\pi ; 2 \pi)$ ?

The positive solvablity of Problem 1 for $p=1$ would solve the aforementioned problem on nontrivial solution of convolution type equations. However, as it will be show later, there exist functions $f \in W_{\sigma}^{1}$, for which the required splitting is impossible. This is why one has to consider the problem with less restrictive conditions, namely, Problem 2.

Problem 2. Whether each function $f \in W_{\sigma}^{p}, 1 \leqslant p \leqslant 2$, admits splitting $f=f_{4}-f_{5}$, where functions $f_{4}$ and $f_{5}$ are analytic in $\mathbb{C}_{+}, f_{4}$ obeys condition $B\left(0 ; \frac{\pi}{2}\right)$ and $f_{5}$ obeys condition $B\left(-\frac{\pi}{2} ; 0\right)$ ?

Here we consider the most interesting from qualitative point of view cases $p=2$ and $p=1$. The formulated problem are also of interest in the theory of integral operators and studies of shift operators.

\section{REPRESENTATIONS}

Statements analogous to Paley-Wiener theorem are also known for the cases $1<p<2$ and $p=1$ (see [16], [17], [22]).

Theorem B1. (Boas R., [23]) Space $W_{\sigma}^{1}$ coincides with the space of functions represented by (1), where the function

$$
\varphi^{*}(t)=\left\{\begin{array}{c}
\varphi(t),|t| \leqslant \sigma \\
0, \quad|t|>\sigma
\end{array}\right.
$$

has an absolutely convergent Fourier series on $(-\sigma-\delta ; \sigma+\delta)$ for some $\delta>0$.

It is well-known (see [5]) that if the Fourier series of function $\varphi^{*}$ converges absolutely on $(-\sigma-\delta ; \sigma+\delta)$ for some positive $\sigma$ and $\delta$, then for each $\delta_{1} \in[0 ;+\infty)$ the Fourier series of function $\varphi^{*}$ converges absolutely on $\left(-\sigma-\delta_{1} ; \sigma+\delta_{1}\right)$.

In another form the criterion for belonging to space $W_{\sigma}^{1}$ was obtained by G. Ber.

Theorem B2. Space $W_{\sigma}^{1}$ coincides with the space of functions represented by (1), where

$$
\varphi(t)=\sum_{k=-\infty}^{+\infty} c_{k} e^{-\frac{i k \pi t}{\sigma}}
$$

at that, $\left(c_{k}\right) \in l^{1}$ and

$$
\sum_{m=-\infty}^{+\infty}\left|\sum_{k=-\infty}^{+\infty}(-1)^{k+m} c_{k+m} \frac{k}{k^{2}+1}\right|<+\infty .
$$

Let us formulate the result of $\mathrm{R}$. Boas in a more convenient for us form by employing PaleyWiener theorem.

Lemma 1. Function $f$ belongs to space $W_{\sigma}^{1}, \sigma>0$, if and only if it can be represented as

$$
f(z)=\sum_{k=-\infty}^{+\infty}(-1)^{k} c_{k} \frac{\pi \sin \sigma z}{\sigma z-\pi k}
$$

where $\left(c_{k}\right) \in l^{2}$ and

$$
\sum_{k=-\infty}^{+\infty}\left|f\left(\frac{k \pi}{\sigma}(1-\delta)\right)\right|<+\infty
$$


for some $\delta \in(0 ; 1)$.

A general description of interpolation sequences in $W_{\sigma}^{p}$ was obtained by A. Beurling, Yu. Lyubarskii, K. Seip [19], [20]. For our purpose the formulated result is sufficient. We note that condition $\left(c_{k}\right) \in l^{1}$ follows from condition (3).

Corollary 1. If $f \in W_{\sigma}^{1}, \sigma>0$, then representation (2) holds true and

$$
\sum_{k=-\infty}^{+\infty}(-1)^{k} c_{k}=0
$$

Indeed, by Theorem B1, $\varphi(\sigma)=0$, but

$$
\varphi(\sigma)=\sum_{k=-\infty}^{+\infty} c_{k} e^{-i k \pi}=\sum_{k=-\infty}^{+\infty}(-1)^{k} c_{k}
$$

Corollary 2. Condition (3) is equivalent to

$$
\sum_{k=-\infty}^{+\infty}\left|\sum_{s=-\infty}^{+\infty}(-1)^{s} c_{s} \frac{\sin (k \pi(1-\delta))}{k(1-\delta)-s}\right|<+\infty
$$

\section{EXPANSIONS in PALEY-WienER SPACE}

For the case $p=2$ we have a simple solution of Problem 1 based on Paley-Wiener theorem:

$$
f_{2}(z)=\frac{1}{\sqrt{2 \pi}} \int_{0}^{\sigma} \varphi(t) e^{i t z} d t, \quad f_{3}(z)=-\frac{1}{\sqrt{2 \pi}} \int_{-\sigma}^{0} \varphi(t) e^{i t z} d t .
$$

But for $p=1$ the above splitting does not solve Problem 1 in the general case. For instance, if $\varphi(t)=\sigma-|t|$, then

but

$$
f(z)=\sqrt{\frac{2}{\pi}} \frac{1-\cos \sigma z}{z^{2}} \in W_{\sigma}^{1}
$$

$$
f_{2}(z)=\frac{1}{\sqrt{2 \pi}} \frac{-e^{i \sigma z}+1+i \sigma z}{z^{2}} \notin W_{\sigma}^{1}, \quad f_{3}(z)=\frac{1}{\sqrt{2 \pi}} \frac{e^{-i \sigma z}-1+i \sigma z}{z^{2}} \notin W_{\sigma}^{1} .
$$

Lemma 2. If for $f \in W_{\sigma}^{1}$ Problem 1 is positively solvable, then under the notations of Paley-Wiener theorem representations (4) are valid.

Proof. As $p=1$, function $f_{2}$ satisfy conditions $A(0 ; \pi)$ and $B(-\pi ; 0)$ that implies $f_{2}(z) e^{-i \sigma z / 2} \in$ $W_{\sigma / 2}^{1}$. Hence, by Paley-Wiener theorem

$$
f_{2}(z)=\frac{1}{\sqrt{2 \pi}} \int_{0}^{\sigma} \varphi_{2}(t) e^{i t z} d t
$$

for some function $\varphi_{2} \in L^{2}[0 ; \sigma]$. In the same way for a function $\varphi_{3} \in L^{2}[-\sigma ; 0]$ we obtain the identity

$$
f_{3}(z)=-\frac{1}{\sqrt{2 \pi}} \int_{-\sigma}^{0} \varphi_{3}(t) e^{i t z} d t
$$

Function $f-f_{2}+f_{3}$ belongs to space $W_{\sigma}^{2}$ and thus it obeys the representation

$$
f(z)-f_{2}(z)+f_{3}(z)=\frac{1}{\sqrt{2 \pi}} \int_{-\sigma}^{\sigma} \widetilde{\varphi}(t) e^{i t z} d t
$$


for some function $\widetilde{\varphi} \in L^{2}[-\sigma ; \sigma]$. But $f-f_{2}+f_{3}$ is identically zero, and thus $\widetilde{\varphi} \equiv 0$. It implies the statement of the lemma.

We shall provide two solvability criteria for Problem 1 basing on Boas and Ber theorems.

Theorem 1. If $f \in W_{\sigma}^{1}$, then Problem 1 is positively solvable if and only if

$$
\sum_{m=-\infty}^{+\infty}\left|\sum_{k=-\infty}^{+\infty} c_{k} \frac{(-1)^{m+k} e^{i \pi \delta m}-1}{m-\delta m-k}\right|<+\infty
$$

for some $\delta \in(0 ; 1)$. At that we suppose that

$$
\frac{(-1)^{m+k} e^{i \pi \delta m}-1}{m-\delta m-k}=\pi i, \quad \text { if } \quad m-\delta m-k=0 .
$$

Proof. If $f \in W_{\sigma}^{1}$, it obeys representation (1). By Lemma 2, Problem 1 is positively solvable if and only if function $f_{2}$ defined by the first identity in (4) belongs to $W_{\sigma}^{1}$. Function $f_{2}$ satisfies the representation

$$
\begin{aligned}
f_{2}(z) & =\frac{1}{\sqrt{2 \pi}} \int_{0}^{\sigma} \sum_{k=-\infty}^{+\infty} c_{k} e^{-\frac{i k \pi t}{\sigma}} e^{i t z} d t=\frac{1}{\sqrt{2 \pi}} \sum_{k=-\infty}^{+\infty} c_{k} \int_{0}^{\sigma} e^{i t\left(z-\frac{k \pi}{\sigma}\right)} d t \\
& =\frac{1}{\sqrt{2 \pi}} \sum_{k=-\infty}^{+\infty} c_{k} \frac{e^{i \sigma(z-k \pi / \sigma)}-1}{i(z-k \pi / \sigma)} .
\end{aligned}
$$

We note that

$$
\sqrt{2 \pi} f_{2}\left(\frac{m \pi}{\sigma}(1-\delta)\right)=\sum_{k=-\infty}^{+\infty} c_{k} \frac{e^{i \sigma\left(\frac{m \pi}{\sigma}(1-\delta)-\frac{k \pi}{\sigma}\right)}-1}{i\left(\frac{m \pi}{\sigma}(1-\delta)-\frac{k \pi}{\sigma}\right)}=\sum_{k=-\infty}^{+\infty} c_{k} \frac{\sigma e^{i \pi(m-\delta m-k)}-1}{i \pi(m-\delta m-k)}
$$

that implies condition (5). By Paley-Wiener theorem, $f_{2} \in W_{\sigma}^{2}$ and hence, the sequence of the coefficients in expansion (2) belongs to $l^{2}$. It remains to apply Lemma 1 to $f_{2}$.

Theorem 2. If $f \in W_{\sigma}^{1}$, then Problem 1 is positively solvable if and only if

$$
\begin{gathered}
\sum_{m=-\infty}^{+\infty}\left|\sum_{k=-\infty}^{+\infty} \frac{k}{k^{2}+1} \sum_{s=-\infty}^{+\infty} c_{s} \frac{(-1)^{s}-(-1)^{k+m}}{k+m-s}\right|<+\infty \\
\quad \sum_{m=-\infty}^{+\infty}\left|\sum_{k=-\infty}^{+\infty} c_{k} \frac{(-1)^{k+m}-1}{m-k}\right|<+\infty
\end{gathered}
$$

at that we suppose that

$$
\frac{(-1)^{s}-(-1)^{k+m}}{k+m-s}=\pi i, \quad \text { if } s=k+m, \text { and } \quad \frac{(-1)^{k+m}-1}{m-k}=\pi i, \quad \text { if } m=k .
$$

Proof. In the proof of the previous theorem it was shown that

$$
f_{2}(z)=\frac{1}{\sqrt{2 \pi}} \sum_{k=-\infty}^{+\infty} c_{k} \frac{(-1)^{k} e^{i \sigma z}-1}{i(z-k \pi / \sigma)} .
$$

We apply Theorem B2 for $f_{2}$ writing its conditions as

$$
\sum_{m=-\infty}^{+\infty}\left|\sum_{k=-\infty}^{+\infty}(-1)^{k+m} f\left(\frac{k+m}{\sigma}\right) \frac{k}{k^{2}+1}\right|<+\infty
$$


Then

$$
\sum_{m=-\infty}^{+\infty}\left|\sum_{k=-\infty}^{+\infty} \frac{(-1)^{k+m} k}{k^{2}+1} \sum_{s=-\infty}^{+\infty} c_{s} \frac{(-1)^{s} e^{i \sigma \frac{k+m}{\sigma} \pi}-1}{i\left(\frac{k+m}{\sigma} \pi-\frac{s \pi}{\sigma}\right)}\right|<+\infty
$$

that yields condition (6). Since

$$
f\left(\frac{\pi m}{\sigma}\right)=\frac{1}{\sqrt{2 \pi}} \sum_{k=-\infty}^{+\infty} c_{k} \frac{(-1)^{k} e^{i \pi m}-1}{i(\pi m / \sigma-k \pi / \sigma)}
$$

the belonging of sequence of the coefficients in expansion (2) for function $f_{2}$ to space $l^{1}$ we obtain condition (7).

The obtained conditions are rather complicated while checking them for particular functions. Quite often it is more convenient to employ simpler necessary or sufficient conditions.

Corollary 3. If for a function $f \in W_{\sigma}^{1}$ Problem 1 is positively solvable, its coefficients in expansion (2) satisfy

$$
\sum_{k=-\infty}^{+\infty} c_{k}=0
$$

Indeed, if $f_{2}$ solves Problem 1, representation (4) holds true, and by Theorem B1, $\varphi(0)=$ $\varphi(\sigma)=0$. But $\varphi(0)=\sum_{k=-\infty}^{+\infty} c_{k} e^{0}=\sum_{k=-\infty}^{+\infty} c_{k}$. It follows easily that for the function

$$
f(z)=\frac{1-\cos \sigma z}{z^{2}} \in W_{\sigma}^{1}
$$

Problem 1 is unsolvable since condition (8) fails:

$$
\sum_{k=-\infty}^{+\infty}(-1)^{k} f\left(\frac{k \pi}{\sigma}\right)>0
$$

Theorem 3. If in representation (2) of function $f \in W_{\sigma}^{1}$ coefficients $c_{k}$ vanish for all odd $k \in \mathbb{Z}$, Problem 1 is positively solvable for this function.

Proof. Let us show that the desired splitting reads as

$$
f_{2}(z)=\frac{1}{2 i} \sum_{k=-\infty}^{+\infty} \frac{c_{2 k}\left(e^{i \sigma z}-1\right)}{z-2 k \pi / \sigma}, \quad f_{3}(z)=\frac{1}{2 i} \sum_{k=-\infty}^{+\infty} \frac{c_{2 k}\left(e^{-i \sigma z}-1\right)}{z-2 k \pi / \sigma}
$$

Indeed, $f_{2}$ and $f_{3}$ are entire functions of exponential type $\leqslant \sigma$. We observe that

$$
f_{2}(z)=\frac{f(z)\left(e^{i \sigma z}-1\right)}{2 i \sin \sigma z}=\frac{f(z) e^{i \sigma z / 2}}{2 \cos (\sigma z / 2)},
$$

and hence function $f_{2}^{*}(z)=f_{2}(z+i)$ belongs to $L^{1}(\mathbb{R})$. Thus, by the definition, $f_{2}^{*}(z) e^{-i \sigma z} \in W_{\sigma}^{1}$ that implies $f_{2}(z) e^{-i \sigma z} \in W_{\sigma}^{1}$. In the same way one can show that $f_{3} e^{i \sigma z} \in W_{\sigma}^{1}$.

\section{EXPANSIONS IN WEIGHTED HARDY SPACE}

Let us formulate a corollary of one of the results by B. Vinnitskii [6].

Theorem C. Space $H_{\sigma}^{2}\left(\mathbb{C}_{+}\right)$coincides with the space of functions represented as

$$
f(z)=\frac{1}{\sqrt{2 \pi}} \int_{\partial D_{\sigma}} \varphi(t) e^{t z} d t,
$$

where $\varphi \in L^{2}\left[\partial D_{\sigma}\right], D_{\sigma}=\{z:|\operatorname{Im} z|<\sigma, \operatorname{Re} z<0\}$. 
This statement implies the following solution to Problem 2 in the case $p=2$ :

$$
f_{4}(z)=\frac{1}{\sqrt{2 \pi}} \int_{\partial D_{\sigma} \cap \mathbb{C}^{+}} \varphi(t) e^{t z} d t, \quad f_{5}(z)=-\frac{1}{\sqrt{2 \pi}} \int_{\partial D_{\sigma} \cap \mathbb{C}^{-}} \varphi(t) e^{t z} d t,
$$

where $\mathbb{C}^{+}=\{z: \operatorname{Im} z>0\}, \mathbb{C}^{-}=\{z: \operatorname{Im} z<0\}$.

Remark 1. For the function

$$
f(z)=\frac{1-\cos \sigma z}{z^{2}} \in W_{\sigma}^{1}
$$

Problem 2 is solvable. Indeed,

$$
f_{4}(z)=-\frac{1}{\sqrt{2 \pi}} \frac{e^{i \sigma z}-1-i \sigma z+\frac{i \sigma}{z+\pi} z^{2}}{z^{2}}, \quad f_{5}(z)=-\frac{1}{\sqrt{2 \pi}} \frac{e^{-i \sigma z}-1+i \sigma z-\frac{i \sigma}{z+\pi} z^{2}}{z^{2}} .
$$

We denote by $\widehat{H}_{\sigma}^{1}\left(\mathbb{C}_{+}\right)$the set of all possible products $f_{1} f_{2}$, where $f_{1} \in H_{\sigma / 2}^{2}\left(\mathbb{C}_{+}\right), f_{2} \in$ $H_{\sigma / 2}^{2}\left(\mathbb{C}_{+}\right)$. It is obvious that $\widehat{H}_{\sigma}^{1}\left(\mathbb{C}_{+}\right) \subset H_{\sigma}^{1}\left(\mathbb{C}_{+}\right)$. We do not know whether space $\widehat{H}_{\sigma}^{1}\left(\mathbb{C}_{+}\right)$ coincides with $H_{\sigma}^{1}\left(\mathbb{C}_{+}\right)$. The positive solvability of Problem 2 implies a more general result.

Lemma 3. If Problem 2 is positively solvable for $W_{\sigma}^{1}$, it is positively solvable for each function in space $\widehat{H}_{\sigma}^{1}\left(\mathbb{C}_{+}\right)$.

Proof. By Theorem C, if $f_{1} \in H_{\sigma / 2}^{2}\left(\mathbb{C}_{+}\right)$, then $f_{1}(z)=e^{\frac{i \sigma z}{z}} h_{1}(z)+h_{2}(z)+e^{-\frac{i \sigma z}{z}} h_{3}(z)$, where

$$
\begin{gathered}
h_{1}(z)=\frac{1}{\sqrt{2 \pi}} \int_{-\infty}^{0} f\left(t+i \frac{\sigma}{2}\right) e^{t z} d t, \quad h_{2}(z)=-\frac{i}{\sqrt{2 \pi}} \int_{\frac{\sigma}{2}}^{\frac{\sigma}{2}} f(i t) e^{i t z} d t, \\
h_{3}(z)=\frac{1}{\sqrt{2 \pi}} \int_{0}^{-\infty} f\left(t-i \frac{\sigma}{2}\right) e^{t z} d t .
\end{gathered}
$$

In accordance with Paley-Wiener theorems (see above and [15]), we have $h_{1} \in H^{2}\left(\mathbb{C}_{+}\right), h_{2} \in$ $W_{\frac{\sigma}{2}}^{2}, h_{3} \in H^{2}\left(\mathbb{C}_{+}\right)$. In the same way, if $f_{1}^{*} \in H_{\frac{\sigma}{2}}^{2}$, then $f_{1}^{*}(z)=e^{\frac{i \sigma z}{2}} h_{1}^{*}(z)+h_{2}(z)+e^{-\frac{i \sigma z}{2}} h_{3}^{*}(z)$, where $h_{1}^{*} \in H^{2}\left(\mathbb{C}_{+}\right), h_{2}^{*} \in W_{\frac{\sigma}{2}}^{2}, h_{3}^{*} \in H^{2}\left(\mathbb{C}_{+}\right)$. Conditions $A\left(-\frac{\pi}{2} ; 0\right)$ and $B\left(0 ; \frac{\pi}{2}\right)$ hold true for the functions $e^{\frac{i \sigma z}{2}} h_{1}(z) f_{1}^{*}(z)+h_{2}(z) e^{\frac{i \sigma z}{2}} h_{1}^{*}(z)$, while conditions $A\left(0 ; \frac{\pi}{2}\right)$ and $B\left(-\frac{\pi}{2} ; 0\right)$ are satisfied for the function $e^{-\frac{i \sigma z}{2}} h_{3}(z) f_{1}^{*}(z)+h_{2}(z) e^{-\frac{i \sigma z}{2}} h_{3}^{*}(z) p=1$. Thus, Problem 2 is positively solvable for the function $f_{1} f_{1}^{*}$ if and only if the same is true for the function $h_{1} h_{1}^{*}$.

Theorem 4. If representation (2) for a function $f \in W_{\sigma}^{1}$ involves only finitely many nonzero coefficients $c_{k}$, then Problem 2 is positively solvable for this function.

Proof. For the sake of simplifying the arguing, we assume that $\sigma=\pi$. The hypothesis of the theorem implies

$$
f(z)=\sin \pi z\left(\frac{(-1)^{k_{1}} c_{k_{1}}}{z-k_{1}}+\frac{(-1)^{k_{2}} c_{k_{2}}}{z-k_{2}}+\ldots+\frac{(-1)^{k_{n}} c_{k_{n}}}{z-k_{n}}\right), \quad k_{n} \in Z .
$$

By Corollary 1, the function in the brackets is rational and after reducing to the common denominator the degree of the latter exceeds that of the numerator at least by two. We let

$$
\begin{aligned}
& f_{4}(z)=\frac{e^{i \pi z}-\frac{a_{n-1} z^{n-1}+\ldots+a_{1} z+a_{0}}{(z+1)^{n-1}}}{2 i}\left(\frac{(-1)^{k_{1}} c_{k_{1}}}{z-k_{1}}+\frac{(-1)^{k_{2}} c_{k_{2}}}{z-k_{2}}+\ldots+\frac{(-1)^{k_{n}} c_{k_{n}}}{z-k_{n}}\right), \\
& f_{5}(z)=\frac{e^{-i \pi z}-\frac{a_{n-1} z^{n-1}+\ldots+a_{1} z+a_{0}}{(z+1)^{n-1}}}{2 i}\left(\frac{(-1)^{k_{1}} c_{k_{1}}}{z-k_{1}}+\frac{(-1)^{k_{2}} c_{k_{2}}}{z-k_{2}}+\ldots+\frac{(-1)^{k_{n}} c_{k_{n}}}{z-k_{n}}\right),
\end{aligned}
$$


where $a_{0}, \ldots, a_{n-1}$ are unknown coefficients. We choose them as solutions to the system of equations

$$
e^{ \pm i \pi z}-\left.\frac{a_{n-1} z^{n-1}+\ldots+a_{1} z+a_{0}}{(z+1)^{n-1}}\right|_{z=k_{1}, \ldots, k_{n}}=0
$$

The equivalent system is

$$
\cos \pi z(z+1)^{n-1}-a_{n-1} z^{n-1}-\ldots-a_{1} z-\left.a_{0}\right|_{z=k_{1}, \ldots, k_{n}}=0 .
$$

We write it as

$$
\left\{\begin{array}{c}
a_{0}+a_{1} k_{1}+a_{2} k_{1}^{2}+\ldots+a_{n-1} k_{1}^{n-1}=(-1)^{k_{1}}\left(k_{1}+1\right)^{n-1} \\
a_{0}+a_{1} k_{2}+a_{2} k_{2}^{2}+\ldots+a_{n-1} k_{2}^{n-1}=(-1)^{k_{2}}\left(k_{2}+1\right)^{n-1} \\
\ldots \\
a_{0}+a_{1} k_{n}+a_{2} k_{n}^{2}+\ldots+a_{n-1} k_{n}^{n-1}=(-1)^{k_{n}}\left(k_{n}+1\right)^{n-1} .
\end{array}\right.
$$

The determinant of this system is Vandermondian

$$
\left|\begin{array}{cccc}
1 & k_{1} & \cdots & k_{1}^{n-1} \\
1 & k_{2} & \cdots & k_{2}^{n-1} \\
\vdots & \vdots & \ddots & \vdots \\
1 & k_{n} & \cdots & k_{n}^{n-1}
\end{array}\right|=\prod_{1 \leqslant m<s \leqslant n}\left(k_{m}-k_{s}\right) \neq 0
$$

hence, there exists the unique solution to the above system. Thus, functions $f_{4}$ and $f_{5}$ solve Problem 2.

In view of the above theorem there appears a question on possibility of applying this method for the case, when $f$ has infinitely mane non-zero coefficients. That is, the problem is to find a function $h \in H^{\infty}\left(\mathbb{C}_{+}\right), p \geqslant 1$, such that $e^{i \pi z}-\left.h(z)\right|_{z \in N \cup\{0\}}=0$ instead of

$$
\frac{a_{n-1} z^{n-1}+\ldots+a_{1} z+a_{0}}{(z+1)^{n-1}} \text {. }
$$

Indeed, if $f_{1}(z)=e^{-\frac{i \pi z}{2}}\left(e^{i \pi z}-h(z)\right)$, then $f_{1} \in H_{\pi / 2}^{\infty}$ and the zero sets of functions $e^{i \pi z}-h(z)$ and $f_{1}$ coincide. B. Vinnitskii showed [6] that for the sequence of zeroes $\left(\lambda_{n}\right)$ in $\mathbb{C}_{+}$for function $H_{\pi / 2}^{\infty}$ the condition

$$
\limsup _{r \rightarrow+\infty}\left(\sum_{1<\left|\lambda_{n}\right| \leqslant r}\left(\frac{1}{\left|\lambda_{n}\right|}-\frac{\left|\lambda_{n}\right|}{r^{2}}\right) \frac{\operatorname{Re} \lambda_{n}}{\left|\lambda_{n}\right|}-\frac{1}{2} \ln r\right)<+\infty
$$

is necessary and sufficient. But the sequence $\lambda_{n}=n$ does not satisfy this criterion since

$$
\begin{aligned}
\sum_{1<\left|\lambda_{n}\right| \leqslant r}\left(\frac{1}{\left|\lambda_{n}\right|}-\frac{\left|\lambda_{n}\right|}{r^{2}}\right) \frac{\operatorname{Re} \lambda_{n}}{\left|\lambda_{n}\right|} & =\sum_{n=2}^{[r]}\left(\frac{1}{n}-\frac{n}{r^{2}}\right) \frac{n}{n} \\
& =\ln [r]-\frac{[r]([r]+1)}{2} \frac{1}{r^{2}}+O(1)=\ln r+O(1), \quad r \rightarrow+\infty
\end{aligned}
$$

Theorem 5. If in representation (2) for a function $f \in W_{\sigma}^{1}$ coefficients $c_{k}$ vanish for odd $k \in \mathbb{N}$, then Problem 2 is positively solvable.

Proof. Let us show that the desired expansion reads as

$$
f_{4}(z)=\frac{1}{2 i} \sum_{k=-\infty}^{+\infty} \frac{c_{2 k}\left(e^{i \sigma z}-1\right)}{z-2 k \pi / \sigma}, \quad f_{5}(z)=\frac{1}{2 i} \sum_{k=-\infty}^{+\infty} \frac{c_{2 k}\left(e^{-i \sigma z}-1\right)}{z-2 k \pi / \sigma}
$$


As in the proof of Theorem 3, one can show that

$$
f_{4}(z)=\frac{f(z) e^{i \sigma z / 2}}{2 \cos (\sigma z / 2)} .
$$

The statement of the theorem for $f_{4}$ follows from the inequalities [21]

$$
\sup _{y \in \mathbb{R}}\left\{\int_{0}^{+\infty}\left|f_{4}^{*}(x+i y)\right| e^{-\sigma|y|} d x\right\}<+\infty ; \quad \sup _{x>0}\left\{\int_{-\infty}^{+\infty}\left|f_{4}^{*}(x+i y)\right| e^{-\sigma|y|} d y\right\}<+\infty,
$$

where $f_{4}^{*}(z)=f_{4}(z) e^{-i \sigma z / 2}$. The former of them together with

$$
\sup _{y \in \mathbb{R} \backslash[-1 ; 1]}\left\{\int_{0}^{+\infty}\left|f_{4}^{*}(x+i y)\right| e^{-\sigma|y|} d x\right\}<+\infty
$$

are obvious. To prove the condition

$$
\sup _{y \in[-1 ; 1]}\left\{\int_{0}^{+\infty}\left|f_{4}^{*}(x+i y)\right| e^{-\sigma|y|} d x\right\}<+\infty
$$

we note that the factorization theorem [18] for the spaces $H_{\sigma}^{p}\left(\mathbb{C}_{+}\right)$implies the existence of $c \in \mathbb{R}$ such that $f_{4}^{*}(z) e^{-c z} \in H_{\sigma}^{1}\left(\mathbb{C}_{+}\right)$. This is why to complete the proof it remains to employ a Phrágmen-Lindelöf type theorem for the half-strip. The statement on $f_{5}$ can be checked in the same way.

It is obvious that similar result is true once instead of vanishing the coefficients with odd indices we can assume the same for the even indices. In view of Theorem 4, the solvability conditions for Problem 2 given in Theorem 5, can be weakened. One can assume that only finitely many with either odd or even indices are non-zero.

\section{BIBLIOGRAPHY}

1. B.J. Levin and Ju.I. Ljubarskiu Interpolation by means of special classes of entire functions and related expansions in series of exponentials // Izv. AN SSSR. Ser. matem. 39:3, 657-702 (1975). [Math. USSR-Izv. 9:3, 621-662 (1975).]

2. R.E.A.C. Paley, N. Wiener. Fourier transforms in complex domain. AMS, Providence, RI (1934).

3. R.S. Yulmukhametov. Splitting entire functions with zeros in a strip // Matem. Sbornik. 186:7, 147-160 (1995). [Sb. Math. 186:7, 1071-1084 (1995).]

4. R.S. Yulmukhametov. Solution of the Ehrenpreis factorization problem // Matem. Sbornik. 190:4, 123-157 (1995). [Sb. Math. 190:4, 597-629, (1999).]

5. N.K. Bari. A treatise on trigonometric series. Fizmatgiz, Moscow (1961). [Pergamon Press, N.Y. (1964).]

6. B.V. Vinnitskii. On zeros of functions analytic in a half plane and completeness of systems of exponents // Ukr. Matem. Zhurn. 46:5, 484-500 (1994). [Ukr. Math. J. 46:5, 514-532 (1994).]

7. B.V. Vinnitskii, V.M. Dilnyi. On analogue of Paley-Wiener theorem for weighted Hardy spaces // Matematychni Studii. 14:1, 35-40 (2000). (in Ukrainian).

8. B.V. Vinnitskii, V. N. Dil'nyi. A generalization of the Beurling-Lax theorem // Matem. Zametki. 79:3, 362-368 (2006). [Math. Notes. 79:3-4, 335-341 (2006).]

9. P. Koosis. Introduction to $H_{p}$ spaces. Cambridge Univ. Press, Cambridge (1999).

10. A.M. Sedleckiu. An equivalent definition of $H^{p}$ spaces in the half-plane and some applications // Matem. Sbornik. 96:1, 75-82 (1975). [Math. USSR-Sbornik. 25:1, 69-76 (1975).]

11. P. Lax. Translation invariant subspaces // Acta math. 101:3-4, 163-178 (1959).

12. N.K. Nikolskii. Treatise on the shift operator. Spectral function theory. Springer-Verlag, Berlin (1986). 
13. V.P. Gurari1. Harmonic analysis in spaces with weight // Tr. Mosk. Mat. Obs. 35, 21-76 (1976). (in Russian).

14. V. Dilnyi. On cyclic functions in weighted Hardy spaces // Zh. Mat. Fiz. Anal. Geom. 7:1, 19-33 (2011).

15. J.B. Garnett. Bounded analytic functions. Academic Press, N.Y. (1981).

16. B.Ja. Levin Lectures on entire functions. AMS, Providence, RI: Translations of Mathematical Monographs, 150 (1996).

17. G.Z. Ber. On interferention phenomenon in integral metric and approximation of entire functions of exponential type // Teor. Funktsii, Funkts. Anal. Pril. 34, 11-24 (1980). (in Russian).

18. B.V. Vinnitskii, V.M. Dilnyi. On necessary existence conditions for solutions of convolution type equation // Matematychni Studii. 16:1, 61-70 (2001).

19. Y. Lyubarskii, K. Seip. Complete interpolating sequences for Paley-Wiener spaces and Muckenhoupt's $\left(A_{p}\right)$ condition // Rev. Matem. Iberoamer. 13:2, 361-376 (1997).

20. A. Beurling. The collected works of Arne Beurling. Vol. 2. Harmonic analysis. Birkhauser, Boston (1989).

21. V.M. Dil'nyi. Equivalent definition of some weighted Hardy spaces // Ukr. Math. Journ. 60:9, 1477-1482 (2008).

22. L.S. Maergois. An analog of the Paley-Wiener theorem for entire functions of the space $W_{\sigma}^{p}$, $1<p<2$, and some Applications // Comp. Meth. Func. Theor. 6:2, 459-469 (2006).

23. R. Boas. Entire functions. Academic Press, N.Y. (1954).

24. V.M. Dilnyi. On solutions of homogeneous convolution equation in one class of Hardy-Smirnov functions // Matematychni Studii. 14:2, 171-174 (2000). (in Ukrainian).

25. Yu.I. Lyubarskii. Representation of functions in $H^{p}$ on half-plane and some applications // Teor. Funktsii, Funkts. Anal. Pril. 38, 76-84 (1982). (in Russian).

Vladimir Nikolaevich Dilnyi,

Ivan Franko National University of Lviv,

Universitetskaya str., 1,

82000, Lviv, Ukraine

E-mail: dilnyi@mail.ru 